\title{
ARCA: An Algorithm for Mining Association Rules based Concept Lattice
}

\author{
Qing Xia \\ Library \\ Huaihai Institute of Technology \\ Lianyungang, China, 222005 \\ Email:xiaqing1@163.com
}

\author{
Sujing Wang \\ College of Computer Science \\ and Technology, \\ Jilin University, Changchun, China, 130012 \\ Email: sujingwang@ hotmail.com
}

\author{
Zhen Chen \\ College of Computer Science \\ and Technology, \\ Jilin University, Changchun, China, 130012 \\ Email: chenzhen@jlu.edu.cn
}

\author{
Tao Lv \\ Weapon System Engineering Center \\ Artillery Command Academy,XuanHua, China 075100 \\ Email: lvtom@sohu.com
}

\author{
Dongjing Wang \\ Shizhuang Middle School, Lianyungang, China, 222200 \\ Email: rpcwangdongjing@163.com
}

\begin{abstract}
Association rule discovery is one of kernel tasks of data mining. Concept lattice, induced from a binary relation between objects and features, is a very useful formal analysis tool. It represents the unification of concept intension and extension. It reflects the association between objects and features, and the relationship of generalization and specialization among concepts. There is a one-to-one correspondence between concept intensions and closed frequent itemsets. This paper presents an efficient algorithm for mining association rules based concept lattice called Arca (Association Rule based Concept lAttice). Arca algorithm uses concept-matrix to build a part of concept lattice, in which the intension of every concept be put into one-to-one correspondence with a closed frequent itemset. Then all association rules are discovered by 4 operators which are defined in this paper performed on these concepts.
\end{abstract}

Index Terms-Concept lattice, rank of matrix, formal concept analysis.

\section{INTRODUCTION}

Association rule mining from a transaction database has been a very active research area since the publication of the Apriori algorithm [1]. Several improvements to the basic algorithm and many new approaches [2]-[10] have been proposed during the last decade. With the development of research, Association rule discovery is one of kernel tasks of data mining.

Formal Concept Analysis (FCA) was developed by Pro. Wille in 1982 [11]. Concept Lattice, the core data structure in Formal Concept Analysis, has been widely in machine learning, data mining and knowledge discovery, etc. Every node of concept lattice is a formal concept consisting of extent and intent. Concept lattice embodies the relations between extension and intension. Here is a one-to-one correspondence between concept intensions and closed frequent itemsets.

There are various algorithms [12]-[16] of association rule mining using concept lattice. However, These algorithms need to build a complete concept lattice. Based on CMCG algorithm [17], this paper presents an algorithm Arca of association rule mining using a part of concept lattice.
The paper is organized as follows. Section 2 recalls basic definitions of association rule and concept lattice. Section 3 discusses Arca algorithm and four operator. Section 4 gives an experimental evaluation on the time spent of Arca algorithm and Apriori algorithm. Section 5 concludes the paper.

\section{THE DEFINES OF ASSOCIATION RULE AND CONCEPT LATTICE}

Let $\mathscr{I}=\left\{i_{1}, i_{2}, \ldots, i_{m}\right\}$ be a set of $m$ items.Let $(T)=$ $\left\{t_{1}, t_{2}, \ldots, t_{n}\right\}$, the task-relevant data, be a set of database transactions where each transaction $t$ is a set of items such that $t \subseteq \mathscr{I}$. Each transaction is associated with an identifier, called $T I D$. Each transaction $t$ consists of a set of items $I$ from $\mathscr{I}$. If $|I|=k$, then $I$ is called a $k$-itemset. A transaction $t$ is said to contain $I$ if and only if $I \subseteq t$. An association rule is an implication of ten form $I_{1} \Rightarrow I_{2}$, where $I_{1}, I_{2} \subset \mathscr{I}$ and $I_{1} \cap I_{2}=\emptyset$. The rule $I_{1} \Rightarrow I_{2}$ holds in the transaction set $\mathscr{T}$ with support $s$, where $s$ is the percentage of transactions in $\mathscr{T}$ that contain $I_{1} \cup I_{2}$ (i.e., both $I_{1}$ and $I_{2}$ ). This is taken to be the probability, $P\left(I_{1} \cup I_{2}\right)$. The rule $I_{1} \Rightarrow I_{2}$ has confidence $c$ in the transaction set $\mathscr{T}$ if $c$ is the percentage of transactions in $\mathscr{T}$ containing $I_{1}$ that also contain $I_{2}$. This is taken to be the conditional probability, $P\left(I_{2} \mid I_{1}\right)$. That is,

$$
\begin{gathered}
\operatorname{support}\left(I_{1} \Rightarrow I_{2}\right)=P\left(I_{1} \cup I_{2}\right) \\
\operatorname{confidence}\left(I_{1} \Rightarrow I_{2}\right)=P\left(I_{2} \mid I_{1}\right)
\end{gathered}
$$

Given the user defined minimum support minsupp and minimum confidence mincon $f$ thresholds. If the support of $I \subseteq t$ itemset $I$ be greater or equal to minsupp, $I$ is called a frequentitemset.

Example 2.1: For $\mathscr{T}=\{A, B, C, D, E\}, \mathscr{I}=$ $\{A, B, C, D, E\}$, Table I represents a transaction database.

Definition 2.1: A data mining context is a triple: $\mathscr{D}=$ $(\mathscr{T}, \mathscr{I}, \mathscr{R})$, where $\mathscr{I}$ and $\mathscr{T}$ are two sets, and $\mathscr{R}$ is a relation between $\mathscr{I}$ and $\mathscr{T} . \mathscr{T}=\left\{t_{1}, t_{2}, \ldots, t_{n}\right\}$, each $t_{i}(i \leq n)$ is 
TABLE I

A TRANSACTION DATABASE

\begin{tabular}{c|c}
\hline TID & Iterms \\
\hline 1 & $\mathrm{~A} \mathrm{CD}$ \\
2 & $\mathrm{BC} \mathrm{E}$ \\
3 & $\mathrm{ABC} \mathrm{E}$ \\
4 & $\mathrm{~B} \mathrm{E}$ \\
5 & $\mathrm{ABC} \mathrm{E}$ \\
\hline
\end{tabular}

TABLE II

A TRANSACTION DATABASE

\begin{tabular}{cccccc}
\hline & $\mathrm{A}$ & $\mathrm{B}$ & $\mathrm{C}$ & $\mathrm{D}$ & $\mathrm{E}$ \\
\hline 1 & 1 & 0 & 1 & 1 & 0 \\
2 & 0 & 1 & 1 & 0 & 1 \\
3 & 1 & 1 & 1 & 0 & 1 \\
4 & 0 & 1 & 0 & 0 & 1 \\
5 & 1 & 1 & 1 & 0 & 1 \\
\hline
\end{tabular}

$\begin{array}{lllll}A & B & C & D & E\end{array}$

1
2
5
5 $\left(\begin{array}{lllll}1 & 0 & 1 & 1 & 0 \\ 0 & 1 & 1 & 0 & 1 \\ 1 & 1 & 1 & 0 & 1 \\ 0 & 1 & 0 & 0 & 1 \\ 1 & 1 & 1 & 0 & 1\end{array}\right)$

Fig. 1. A context-matrix of the data mining context showed in TableII

called an object. $\mathscr{I}=\left\{i_{1}, i_{2}, \ldots, i_{m}\right\}$, each $i_{j}(j \leq m)$ is called an attribute.

In a data mining context $\mathscr{D}=(\mathscr{T}, \mathscr{I}, \mathscr{R})$, if $(t, i) \in \mathscr{R}$, we say that the attribute $i$ is an attribute of the object $t$, or that $t$ verifies $i$. In this paper, $(t, i) \in \mathscr{R}$ is denoted by 1 , and $(t, i) \notin(R)$ is denoted by 0 . Thus, a data mining context can be represented by a matrix only with 0 and 1 . We say that the matrix is the context-matrix of $\mathscr{D}$.

Example 2.2: Table II represents a data mining context corresponding with the transaction database showed in Table I.

Example 2.3: Fig 1 a context-matrix of the data mining context showed in Table II.

Definition 2.2: Let $\mathscr{D}=(\mathscr{T}, \mathscr{I}, \mathscr{R})$ be a data mining context. We define a function $f(T)$ that produces the set of their common attributes for every set $T \subseteq \mathscr{T}$ of objects to know which attributes from $\mathscr{I}$ are common to these entire objects: $f(T)=\{i \in \mathscr{I} \mid \forall t \in \mathscr{T},(t, i) \in \mathscr{R}\}$

Dually, we define $Y$ for subset of attributes $I \subset \mathscr{I}, g(I)$ denotes the set consisting of those objects in $\mathscr{T}$ that have all the attributes from $\mathscr{I}: g(I)=\{t \in \mathscr{T} \mid \forall i \in \mathscr{I},(t, i) \in \mathscr{R}\}$. Let $h(I)=f(g(I))$.

These two functions are used to determine a formal concept.

Definition 2.3: Let $\mathscr{D}=(\mathscr{T}, \mathscr{I}, \mathscr{R})$ be a data mining context. A pair $(T, I)$ is called a formal concept of $\mathscr{D}$, for short, a concept, if and only if $T \subseteq \mathscr{T}, I \subseteq \mathscr{I}, f(T)=I$ and $g(I)=T$. $T$ is called extent, $I$ is called intent.

Definition 2.4: Let $\mathscr{D}=(\mathscr{T}, \mathscr{I}, \mathscr{R})$ be a data mining

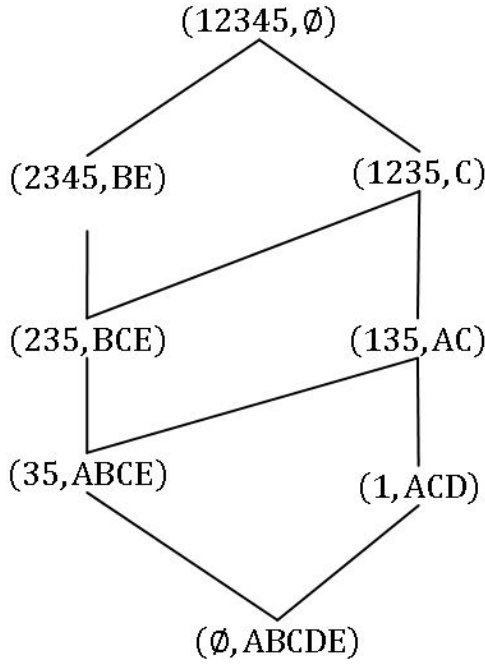

Fig. 2. A Concept lattice for the context of TableII

context. The set of all concepts of $\mathscr{D}$ is denoted by $B(\mathscr{D})$, $C_{1}=\left(T_{1}, I_{1}\right)$ and $C_{2}=\left(T_{2}, I_{2}\right)$ are two concepts in $B(\mathscr{D})$. An partial ordering relation $(\mathrm{i})$ is defined on $\mathrm{B}(\mathrm{D})$ by: $C_{1}<C_{2} \Leftrightarrow T_{1} \subset T_{2}$ or $C_{1}<C_{2} \Leftrightarrow I_{1} \supset I_{2}$

We say that $C_{2}$ is called a superconcept of $C_{1}$ and $C_{1}$ is called a subconcept of $C_{2} . B(\mathscr{D})$ and the partial ordering relation $(i)$ form a compete lattice called the concept lattice of $\mathscr{D}$ and denoted by $L(\mathscr{D})$.

Example 2.4: Fig 2 a concept lattice for the context of Table II.

Definition 2.5: Let $\mathscr{D}=(\mathscr{T}, \mathscr{I}, \mathscr{R})$ be a data mining context. $C_{1}$ and $C_{2}$ are two concepts in $B(\mathscr{D})$. If $C_{1}<C_{2}$ and there is no concept $C_{3}$ in $B(\mathscr{D})$ fulfilling $C_{1}<C_{3}<C_{2}$, $C_{1}$ is called a lower neighbor of $C_{2}$, denoted by $C_{1} \prec C_{2}$, and $C_{2}$ is called a upper neighbors of $C_{1}$.

The set of all lower neighbors of a given concept is a subset of the set consisting of all subconcepts of it.

Definition 2.6: Let $I \subseteq \mathscr{I}$ be a set of items from $\mathscr{D}=$ $(\mathscr{T}, \mathscr{I}, \mathscr{R})$. The support count of the itemset $I$ in $\mathscr{D}$ is:

$$
\operatorname{support}(I)=\frac{|g(I)|}{|\mathscr{T}|}
$$

Definition 2.7: Let $I \subseteq \mathscr{I}$ be a set of items from $\mathscr{D}=$ $(\mathscr{T}, \mathscr{I}, \mathscr{R})$. If support $(I)$ minsupp, I is called a frequent itemset.

Definition 2.8: Let $\mathscr{D}=(\mathscr{T}, \mathscr{I}, \mathscr{R})$ be a data mining context. For given a item $i \in \mathscr{I}$, if the count of $1 \mathrm{~s}$ in the corresponding column of the item $i$ in the concept-matrix of $\mathscr{D}$ is $n$ we say that the rank of item $i$ in concept-matrix of $\mathscr{D}$ is $n$, denoted by $r(i)=n$. If $m=\max \{r(i) \mid i \in \mathscr{I}\}$, we say that the rank of the data mining contgext $\mathscr{D}$ is $m$.

Definition 2.9: Let $\mathscr{D}=(\mathscr{T}, \mathscr{I}, \mathscr{R})$ be a data mining context. The concept-matrix of $C=(T, I)$ is the matrix consisting of these rows what are the corresponding rows of the each element $t$ in set $T$ in context-matrix of $\mathscr{D}$.

Example 2.5: Fig 3 represents the concept-matrix of concept $(135, A C)$. 


$A$
$A$
3
$3\left(\begin{array}{llllll}1 & 0 & 1 & 1 & 0 \\ 1 & 1 & 1 & 0 & 1 \\ 1 & 1 & 1 & 0 & 1\end{array}\right)$

Fig. 3. The concept-matrix of concept $(135, A C)$

Definition 2.10: Let $\mathscr{D}=(\mathscr{T}, \mathscr{I}, \mathscr{R})$ be a data mining context. $C=(T, I)$ is a concept in $B(\mathscr{D})$. If the count of $1 \mathrm{~s}$ in the corresponding column of the item $i$ in the concept-matrix of $C$ is $n$, we say that the rank of item $i$ in concept-matrix of concept $C$ is $n$, denoted by $R_{C}(i)=n$. If $m=\max \left\{R_{C}(i) \mid i \in \mathscr{I}, i \notin I\right\}$, we say that the rank of concept $C$ is $m$.

Property 2.1: The count of objects of subconcept of concept $C$ is equal or lesser than $m$.

Definition 2.11: Let $\mathscr{D}=(\mathscr{T}, \mathscr{I}, \mathscr{R})$ be a data mining context. $C=(T, I)$ is a concept in $B(\mathscr{D})$. Given subset $I_{1} \subseteq \mathscr{I}$, $g_{C}\left(I_{1}\right)$ denotes the set consisting of those transactions in $T$ that have all the itemsets from $\mathscr{I}: g_{C}\left(I_{1}\right)=\{t \in T \mid \forall i \in$ $\left.I_{1},(t, i) \in \mathscr{R}\right\}$

Definition 2.12: Let $\mathscr{D}=(\mathscr{T}, \mathscr{I}, \mathscr{R})$ be a data mining context. $C=(T, I)$ is a concept in $B(\mathscr{D})$.If $|T| \geq|\mathscr{T}| \times$ minsupp. $C$ is called a frequent concept.

Property 2.2: If $C=(T, I)$ is a frequent concept, $I$ is a frequent itemset.

Proof: $|T| \geq|\mathscr{T}| \times$ minsupp, so $\frac{|T|}{|\mathscr{T}|} \geq$ minsupp. And $T=g(I)$, so $\frac{|g(I)|}{|T|} \geq$ minsupp. Then $I$ is a frequent itemset.

Theorem 2.1: Let $\mathscr{D}=(\mathscr{T}, \mathscr{I}, \mathscr{R})$ be a data mining context. $C=(T, I)$ is a concept in $B(\mathscr{D})$. The rank of concept $C$ is $m$. For $\forall i \in\left\{i \mid R_{C}(i)=m, i \in \mathscr{I}\right\}$, $C_{1}=\left(g_{C}(i), f\left(g_{C}(i)\right)\right)$. Then $C_{1}$ is a lower neighbor of $C$.

Proof: By Definition 2.10 And Definition 2.11, we have $\left|g_{C}(i)\right|=m$. Suppose there exist $C_{2}=\left(T_{2}, I_{2}\right)$, where $C_{1} \prec$ $C_{2}$. We can obtain $m=\left|g_{C}(i)\right|<\left|T_{2}\right|<|T|$. By Property 2.1 , we have $\left|T_{2}\right| \leq m$. This result contradicts with $m<\left|T_{2}\right|$. Then $C_{1}$ is a lower neighbor of $C$.

Theorem 2.2: Let $\mathscr{D}=(\mathscr{T}, \mathscr{I}, \mathscr{R})$ be a data mining context. $C=(T, I)$ is a concept in $B(\mathscr{D})$. The rank of concept $C$ is $m . C_{1}=\left(g_{C}\left(i_{1}\right), f\left(g_{C}\left(i_{1}\right)\right)\right)$ is a subconcept of $C$, where $i_{1} \in \mathscr{I}$ and $R_{C}\left(i_{1}\right)=m_{1}>0$. For $\forall C_{2} \in\left\{C_{2}=\right.$ $\left.\left(T_{2}, I_{2}\right)\left|C_{2}<C, m_{1}<\right| T_{2} \mid\right\}$, there NOT exist $g_{C}\left(i_{1}\right) \subset T_{2}$. Then $C_{1}$ is a lower neighbor of $C$.

Proof: Suppose there exist $C_{3}=\left(T_{3}, I_{3}\right)$, where $C_{1}<$ $C_{3}<C$, We have $m_{1}=\left|g\left(i_{1}\right) \cap T\right|<\left|T_{3}\right|<|T|$, it implies that $C_{3} \in\left\{C_{2}=\left(T_{2}, I_{2}\right)\left|C_{2}<C, m_{1}<\right| T_{2} \mid\right\}$, and NOT $g_{C}\left(i_{1}\right) \subset I_{3}$. On the other hand $C_{1}<C_{3}<C$ implies that $g_{C}\left(i_{1}\right) \subset I_{3}$. This result contradicts with NOT $g_{C}\left(i_{1}\right) \subset I_{3}$. Then $C_{1}$ is a lower neighbor of $C$.

Definition 2.13: An association rule is an implication between itemsets of the form $r: I_{1} \rightarrow I_{2}$, where $I_{1}, I_{2} \subset \mathscr{I}$ and $I_{1} \cap I_{2}=\emptyset$. $I_{1}$ is called the antecedent of $r$ and $I_{2}$ is called the consequent of $r$. Below, we define the support and

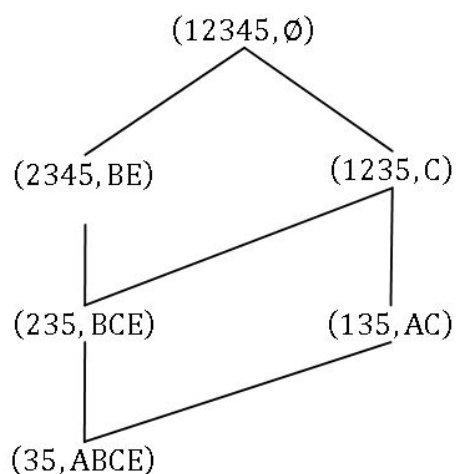

Fig. 4. A concept lattice while minsupp $=0.4$

TABLE III

BASIC ASSOCIATION RULES FROM FIG 4 WITH mincon $f=0.5$

\begin{tabular}{cc}
\hline basic association rule & minimum support \\
\hline$\emptyset \rightarrow B E$ & $4 / 5$ \\
$\emptyset \rightarrow C$ & $4 / 5$ \\
$B E \rightarrow C$ & $3 / 4$ \\
$C \rightarrow B E$ & $3 / 4$ \\
$C \rightarrow A$ & $3 / 4$ \\
$B C E \rightarrow A$ & $2 / 3$ \\
$A C \rightarrow B E$ & $2 / 3$
\end{tabular}

confidence of an association $\mathrm{r}$ :

$$
\begin{gathered}
\text { support }(r)=\frac{\left|g\left(I_{1} \cup I_{2}\right)\right|}{|\mathscr{T}|} \\
\operatorname{confidence}(r)=\frac{\operatorname{support}\left(I_{1} \cup I_{2}\right)}{\operatorname{support}\left(I_{1}\right)}=\frac{\left|g\left(I_{1} \cup I_{2}\right)\right|}{\left|g\left(I_{1}\right)\right|}
\end{gathered}
$$

Mining association rules is to find all rules $r$, where support $(r) \geq \operatorname{minsupp}$ and confidendce $(r) \geq \operatorname{minconf}$.

\section{ARCA ALGORITHM}

When a concept lattice is built, each concept $C=(T, I)$ holds $|T| \geq$ minsupp.

Example 3.1: Fig 4 represents a concept lattice while minsupp $=0.4$.

Definition 3.1: Let $\mathscr{D}=(\mathscr{T}, \mathscr{I}, \mathscr{R})$ be a data mining context. $C_{1}=\left(T_{1}, I_{1}\right)$ and $C_{2}=\left(T_{2}, I_{2}\right)$ are two concepts in $B(\mathscr{D})$ and $C_{1} \prec C_{2}$. If $\frac{\left|T_{1}\right|}{\left|T_{2}\right|} \geq \operatorname{minconf}$, the rule $r: I_{2} \Rightarrow I_{1}-I_{2}$ is called a basic association rules. The minimum support of $r$ is defined as $\frac{\left|T_{1}\right|}{\left|T_{2}\right|}$.

Basic association rules can be mined from concept lattice.

Example 3.2: Table III represents basic association rules from Fig 4 with minconf $=0.5$.

Definition 3.2: Let $r_{1} \equiv I_{1} \rightarrow I_{2}, r_{2} \equiv I_{3} \rightarrow I_{4}$, the minimum supports of $r_{1}$ and $r_{2}$ are con $f_{1}$ and con $f_{2}$, respectively. If $I_{1} \cup I_{2}=I_{3}$, The operator ' + ' can be implemented on $r_{1}$ and $r_{2} . r_{1}+r_{2} \equiv I_{1} \rightarrow I_{4}$. The minimum support of $I_{1} \rightarrow I_{4}$ is defined as con $f_{12}=\operatorname{conf}_{1} \times \operatorname{con} f_{2}$. Let $r_{3} \equiv I_{4} \rightarrow I_{5}$ and its minimum supports is conf $f_{3}$. If $I_{1} \cup I_{2}=I_{3}$ and $I_{3} \cup I_{4}=I_{5}$, the operator ' + ' can be implemented on $r_{1}$, $r_{2}$ and $r_{3} \cdot r_{1}+r_{2}+r_{3} \equiv I_{1} \rightarrow I_{6}$ and its minimum support 
TABLE IV

THE RESULT OF PERFORMING '+' AND ' $\oplus$ ' ON BASIC ASSOCIATION RULES IN TABLE III.

\begin{tabular}{|c|c|c|}
\hline Basic rules & The result of ' + ' & The result of ' $\oplus$ ' \\
\hline & $\emptyset \rightarrow C$ & $\emptyset \rightarrow B C E$ \\
\hline & $B E \rightarrow A$ & $B E \rightarrow A C$ \\
\hline & $\emptyset \rightarrow A$ & $\emptyset \rightarrow A B C E$ \\
\hline & $\emptyset \rightarrow B E$ & $\emptyset \rightarrow B E$ \\
\hline & $C \rightarrow A$ & $C \rightarrow A B E$ \\
\hline & $C \rightarrow A$ & $C \rightarrow A B C E$ \\
\hline & $\emptyset \rightarrow A$ & $\emptyset \rightarrow A C$ \\
\hline & $C \rightarrow B E$ & $C \rightarrow A B E$ \\
\hline & $\emptyset \rightarrow B E$ & $\emptyset \rightarrow A B C E$ \\
\hline
\end{tabular}

is $\operatorname{conf}_{123}=\operatorname{conf}_{1} \times \operatorname{conf}_{2} \times \operatorname{conf}_{3}$. The operator ' + ' can be defined among $\mathrm{n}$ rules by same way.

Definition 3.3: Let $r_{1} \equiv I_{1} \rightarrow I_{2}, r_{2} \equiv I_{3} \rightarrow I_{4}$, the minimum supports of $r_{1}$ and $r_{2}$ are $\operatorname{conf}_{1}$ and $\operatorname{conf}_{2}$, respectively. If $I_{1} \cup I_{2}=I_{3}$, The operator ' $\oplus$ ' can be implemented on $r_{1}$ and $r_{2} . r_{1} \oplus r_{2} \equiv I_{1} \rightarrow I_{2} \cup I_{4}$. The minimum support of $I_{1} \rightarrow I_{2} \cup I_{4}$ is defined as $\operatorname{conf}_{12}=\operatorname{conf}_{1} \times \operatorname{conf}_{2}$. Let $r_{3} \equiv I_{4} \rightarrow I_{5}$ and its minimum supports is conf $f_{3}$. If $I_{1} \cup I_{2}=I_{3}$ and $I_{3} \cup I_{4}=I_{5}$, the operator ' $\oplus$ ' can be implemented on $r_{1}, r_{2}$ and $r_{3} . r_{1} \oplus r_{2} \oplus r_{3} \equiv I_{1} \rightarrow I_{2} \cup I_{4} \cup I_{6}$ and its minimum support is $\operatorname{conf}_{123}=\operatorname{conf}_{1} \times \operatorname{conf} f_{2} \times \operatorname{conf}_{3}$. The operator ' $\oplus$ ' can be defined among $\mathrm{n}$ rules by same way.

Let's examine how to perform ' + ' and ' $\oplus$ ' on basic association rules in Table III Using the following example.

Example 3.3: Since BE, the union of the antecedent and consequent of rule , be not equal to the antecedent of rule , the operators ' + ' and ' $\oplus$ ' cannot be implemented on rule and rule . The antecedent of rule be equal to BE. Therefore, the operators ' + ' and ' $\oplus$ ' cannot be implemented on rule and rule.

$$
\begin{gathered}
+\equiv \emptyset \rightarrow C \\
\oplus \equiv \emptyset \rightarrow B C E
\end{gathered}
$$

Every basic association rule after rule is check whether the operators ' + ' and ' $\oplus$ ' can be implemented on rule and itself. and not. yes. Therefore, we can obtain:

$$
\begin{gathered}
+\quad \equiv B E \rightarrow A \\
+\quad+\quad \equiv \emptyset \rightarrow A \\
\oplus \quad \equiv B E \rightarrow A C \\
\oplus \quad \oplus \quad \equiv \emptyset \rightarrow A B C E
\end{gathered}
$$

Table IV represents the result of performing ' + ' and ' $\oplus$ ' on basic association rules in Table III.

Definition 3.4: Let $r_{1} \equiv I_{1} \rightarrow I_{2}$. The result of performing operator right move is a set of association rules $\left\{I_{1}^{\prime} \rightarrow I_{2} \cup\right.$ $\left.I_{1}^{\prime \prime} \mid I_{1}^{\prime} \cap I_{1}^{\prime \prime}=\emptyset, I_{1}^{\prime} \cup I_{1}^{\prime \prime}=I_{1}, I_{1}^{\prime} \neq, I_{1}^{\prime \prime} \neq \emptyset, g\left(I_{1}\right) \leq g\left(I_{1}^{\prime}\right)\right\}$

Definition 3.5: Let $r_{1} \equiv I_{1} \rightarrow I_{2}$. The result of performing operator decompose is a set of association rule $\left\{I_{1} \rightarrow I_{2}^{\prime} \mid I_{2}^{\prime} \neq\right.$ $\left.\emptyset, I_{2}^{\prime} \subset I_{2}\right\}$
All association rules which confidence be less than 1 are mining out by performing four operators on basic association rules. The pseudo codes are given by Algorithm 1 .

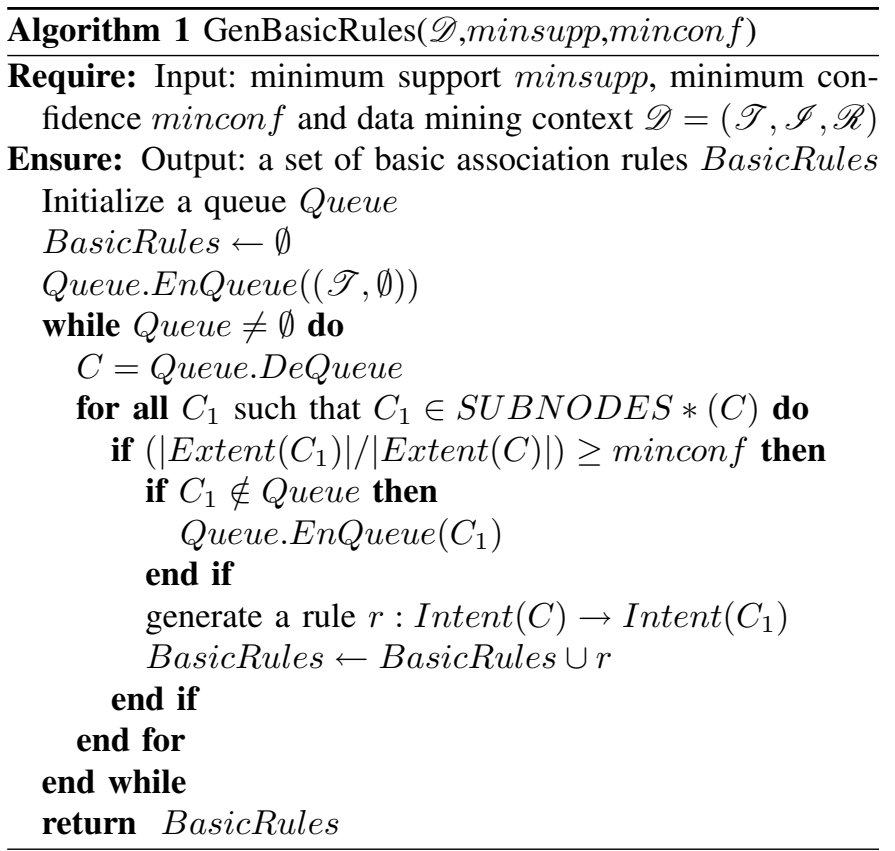

Algorithm 2 SUBNODES*(C, $\mathscr{D}$, minsupp $))$

Require: Input: given a concept $C=(T, I)$, minimum support minsupp, and data mining context $\mathscr{D}=(\mathscr{T}, \mathscr{I}, \mathscr{R})$

Ensure: Output:a set subnodes of concepts which extent's count be greater or equal to minsupp subnodes $\leftarrow \emptyset$

$M \leftarrow$ the concept matrix of concept $C$ compute the rank of every attribute in $M$ $m \leftarrow$ the rank of concept $C$

while $m \geq$ minsupp do

$S \leftarrow$ the set of attributes which ranks equal to $m$ while $S \neq \emptyset$ do

$I_{1} \leftarrow$ the set consisting of a attribute $a$ from $S$ and these attributes from $S$ which corresponding columns are same as column of $a$ in $M$

$S \leftarrow S-I_{1}$

$T_{1} \leftarrow g_{C}\left(I_{1}\right)$

$I_{1} \leftarrow I \cup I_{1}$

if $\forall C_{2}=\left(T_{2}, I_{2}\right) \in$ subnodes, such that NOT $T_{1} \subset$ $T \cap T_{2}$ then subnodes $\leftarrow$ subnodes $\cup\left(T_{1}, I_{1}\right)$

\section{end if}

end while

$m \leftarrow m-1$

end while

return subnodes

Algorithm 1 is to generate basic association rules by build a concept lattice called function SUBNODES* The pseudo codes of this function are given by Algorithm 2 . 


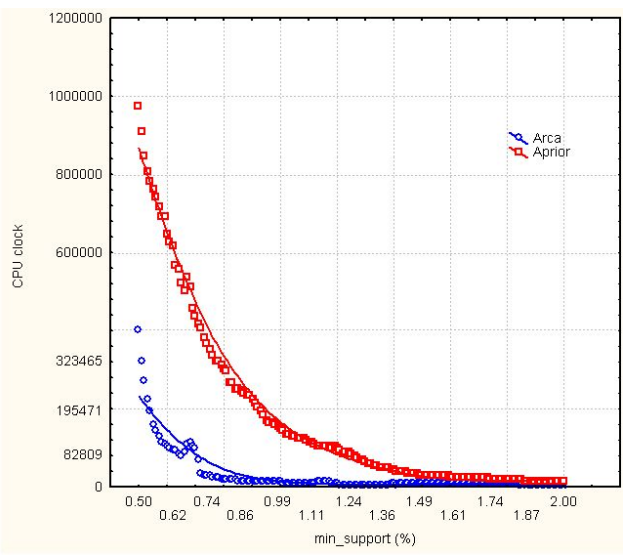

Fig. 5. Running time of algorithms with $\operatorname{mincon} f=0.01$

\section{Evaluation}

In order to evaluate, we implement Algorithm Arca and Algorithm Aprior by Visual C++ and STL. The data set, generated randomly by IBM dataset generator, have 1000 items and 10000 transactions. The result shows that the performance of Arca is as four times higher as Aprior on average. Fig 5 represents Running time of algorithms versus lattice size with minconf $=0.01$.

\section{CONClusion}

Now, there are many algorithms of mining association rules. There is a one-to-one correspondence between concept intensions and closed frequent itemsets. Concept lattice is a good tool for mining association rules.

\section{REFERENCES}

[1] Agrawal R, Imielinski T, Swami A. Mining association rules between sets of items in large databases[J]. ACM SIGMOD Record: 1993, 22: 207-216

[2] Savasere A, Omiecinski E, Navathe S. An efficient algorithm for mining association rules in large databases[C]. Proceedings of the 21st VLDB Conference. the 21st VLDB Conference, Zurich, 1995. 432-444

[3] Park J S, Chen M S, Yu P S. An effective hash-based algorithm for mining association rules[C]. ACM SIGMOD Record . ACM Press, 1995, 24: $175-186$.

[4] Zaki M J, et al. New algorithms for fast discovery of association rules[R]. University of Rochester : 1997.

[5] Shenoy P, et al. Turbo-charging vertical mining of large databases[C]. ACM SIGMOD Record. ACM Press, 2000, . 22-33.

[6] Pasquier N, et al. Discovering frequent closed itemsets for association rules[C]. Proceeding of the 7th International Conference on Database Theory . The 7th International Conference on Database Theory, Jerusalem, 1999. Berlin: Springer-Verlag, 1999, 1540: 398-416.

[7] Pei J, Han J, Mao R. An efficient algorithm for mining frequent closed itemsets[C]. 2000 ACM SIGMOD Workshop on Research Issues in Data Mining and Knowledge Discovery, Dallas, 2000. 21-30.

[8] Han J, et al. FreeSpan: frequent pattern-projected sequential pattern mining $[\mathrm{C}]$. Proceedings of the sixth ACM SIGKDD international conference on Knowledge discovery and data mining. The sixth ACM SIGKDD international conference on Knowledge discovery and data mining, 2000. Boston : ACM Press, 2000, 355-359.

[9] Han J, et al. PrefixSpan: mining sequential patterns efficiently by prefixprojected pattern growth[C]. The Proceedings of 17th International Conference. The 17th International Conference, .2001. 215-224

[10] Han J, Pei J, Yin Y. Mining frequent patterns without candidate generation[C]. 2000 ACM SIGMOD Workshop on Research Issues in Data Mining and Knowledge Discovery, Dallas, 2000. 1-12.
[11] Godin R, Missaoui R. An incremental concept formation approach for learning from databases[J]. Theoretical Computer Science: 1994, 133: 387-419

[12] Valtchev P, Missaoui R, Lebrun P. A partition-based approach towards constructing Galois (concept) lattices[J]. Discrete Mathematics: 2002, 256(3):801-829.

[13] Pasquier N, Bastide Y, Taouil R, Lakhal L. Discovering frequent closed itemsets for association rules[C]. Proceeding of the 7th International Conference on Database Theory. The 7th International Conference on Database Theory, Jerusalem, 1999. Berlin: Springer-Verlag, 1999, 1540: 398-417

[14] Stumme G. Efficient data mining based on formal concept analysis[C]. Proceeding of the 13th International Conference. Database and Expert Systems Applications : 13th International Conference, DEXA 2002 Aixen-Provence, 2002. Berlin: Springer-Verlag, 2453:534-547

[15] Pasquier N, Bastide Y, Taouil R, Lakhal L. Efficient mining of association rules using closed lattices[J]. Information System: Elsevier, 1999, .24(1): 25-46 16. Stumme G, Taouil R, Bastide Y, Pasquier N, Lakhal L. Fast Computation of Concept Lattices Using Data Mining Techniques[C].

[16] Proceeding of 7th Intl. Workshop on Knowledge Representation Meets Databases (KRDB'00). The 7th Intl. Workshop on Knowledge Representation Meets Databases (KRDB'00), Berlin, 2000.

[17] Sujing Wang, Zhen Chen and Dongjing Wang. An Algorithm based on Concept Matrix for Building Concept Lattice with Hasse[C]. Proceeding of 2007 International Conference on Wireless Communications, Networking and Mobile Computing : 2007 International Symposium on Information Systems And Management, Shanghai, 2007. Volume 8, 55935596 